\title{
SM Dermatology Journal
}

\section{NIR Laser Therapy in the Management of Feline Stomatitis}

\author{
Paolo Squarzoni ${ }^{1}$, Daniele Bani ${ }^{2}$, Francesca Cialdai ${ }^{3}$ and Monica Monici ${ }^{3 \star}$ \\ ${ }^{1} M D V$ Freelancer, Bologna, Italy \\ ${ }^{2}$ Department of Clinical and Experimental Medicine, University of Florence, Italy \\ ${ }^{3}$ ASAcampus JL, ASA Research Division. ,Department of Experimental and Clinical Biomedical Sciences \\ "Mario Serio", University of Florence, Italy
}

Article Information

Received date: Nov 24, 2017

Accepted date: Dec 10, 2017

Published date: Dec 15, 2017

\section{*Corresponding author}

Monica Monici, ASAcampus Joint Laboratory, ASA Research Division, Department of Experimental and Clinical Biomedical Sciences "Mario Serio", University of Florence, Viale G. Pieraccini 6, I-50139 Florence, Italy, Tel: +390552758366;

Email: monica.monici@unifi.it

Distributed under Creative Commons CC-BY 4.0

Keywords NIR laser; Laser therapy; Stomatitis

\section{Abstract}

Stomatitis with involvement of the caudal area is a severe inflammation of the oral tissues in cats, which heavily impairs the animal health. The affected gingiva and mucous membrane show different degrees of inflammation, proliferation and ulceration. The only way to manage this serious disease is suppression of inflammation, but often reduction of inflammation at tolerable levels is considered a satisfactory therapeutic result. Many different treatments have been proposed, but currently a therapy of choice and a standardized treatment for stomatitis management have not yet identified. Therefore, new therapeutic strategies are needed to effectively counteract inflammation and improve symptoms.

Laser therapy has been applied with significant results in the management of human patients affected by Oral Mucositis (OM), where it has been effective in reducing inflammation, pain, severity and duration of OM ulcers. The present paper reports the results of a preliminary proof-of-concept clinical study aimed at verifying the hypothesis that NIR laser therapy could be usefully applied to treat cat mucositis.

The enrolled patients were divided into two groups, in order to compare two treatments differing for the energy density released. The effectiveness of laser therapy in reducing inflammation and favoring ulcer healing was assessed by clinical examination of patients, histological analysis and Immunofluorescence microscopy on tissue specimens collected from the animals before and after the cycle of laser therapy.

The outcomes of this preliminary study demonstrate that laser therapy with suitable treatment parameters can be effective in the management of stomatitis.

Even if with different response times in the two groups, laser therapy induced an improvement of the symptoms that was greater and faster in the group treated with lower energy density.

\section{Introduction}

Over the years, feline stomatitis has been classified with various names: feline chronic stomatitis, feline gingivostomatitis, faucitis, Feline Chronic Gingivostomatitis (FCGS), feline stomatitis etc. The last is probably the most appropriate, although some authors use a nomenclature that divides the disease into two types, according to the affected area [1]:

Type 1 - defined as inflammation in areas of oral cavity other than the caudal one.

Type 2 - when the caudal area of the oral cavity is involved (with or without inflammation in other areas of the oral mucosa).

Indeed, type 2 caudal stomatitis is the most severe inflammation of the oral tissues in cats and represents the most difficult variant for therapy. Diagnosis is mainly based on the clinical evaluation, showing inflamed areas with proliferation of gingiva and oral mucosa [2]. In type 2 caudal stomatitis, inflammation and/or proliferation of mucosa of the caudal oral cavity, bounded medially by mouth and palatoglossal folds, dorsally by hard and soft palate and rostrally by alveolar mucosa, is commonly observed.

Caudal stomatitis usually occurs with halitosis, dysphagia, tooth chattering, reluctance to take food, anorexia (complete or partial), algic events during the meal and the yawning, weight loss, opaque mantle and drooling (pseudo-ptyalism). The affected gingiva and mucous membrane show different degrees of inflammation, proliferation and ulceration. Inflammation is often bilateral and symmetrical, the tissues are friable, bright red colored, easily bleeding and with irregular appearance (reminiscent of pebbles).

Feline stomatitis must be differentiated from other oral cavity diseases in the cat, such as juvenile gingivitis, an inflammation that appears immediately after the permanent teeth eruption [3]. This syndrome can be included into two categories: feline hyperplastic gingivitis (in which inflammation is confined to the gingiva), and juvenile periodontitis. Also periodontal disease, the resorptive lesion and systemic diseases that cause damage to mucous membranes should be mentioned. The above diseases may be present in the course of stomatitis, making symptomatology more severe (Figure 1). 


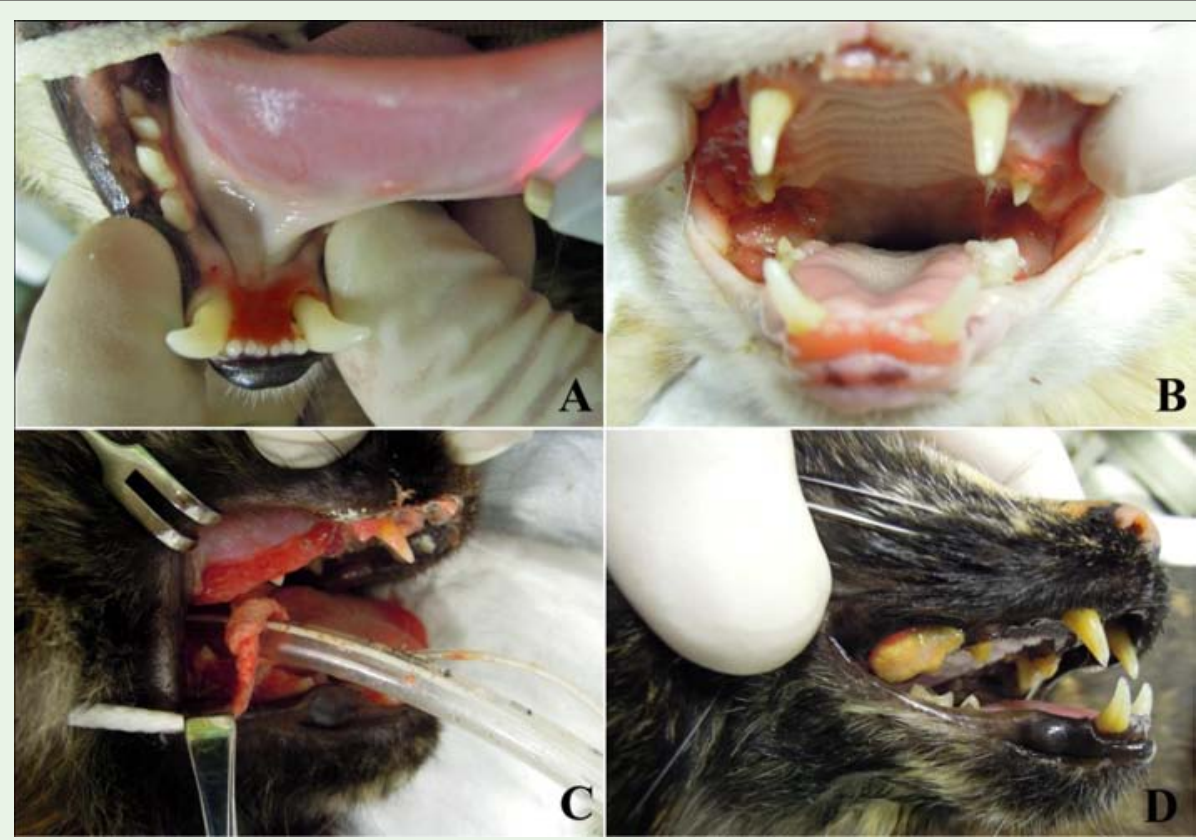

Figure 1: Oral cavity diseases in cats: A) Feline Chronic Gingivo-Stomatitis (FCGS) Type 1, B) FCGS Type 2, C) feline hyperplastic gingivitis, D) periodontal disease.

The etiology of stomatitis is still uncertain. Various etiological agents that create inflammation, either individually or in combination have been identified [1]. Potential causal agents of the anomalous inflammatory response are plaque bacteria, viruses, Bartonella henselae infection, or impaired immune status (FeLV or FIV) $[2,4,5]$. Viruses of the respiratory tract, such as calicivirus (FCV) and herpes virus-1, have been commonly related to some forms of caudal stomatitis [6]. Individually considered, none of these etiologic agents can fully account for the development of the disease [6], while it seems that a combination of several factors may occur, such as viruses, bacteria, altered immune status, environment, race, etc.

The control of inflammation is deemed the major pathway to keep this disease under control. Therefore, suppression of inflammation is the purpose of stomatitis therapy, but often reducing inflammation at tolerable levels for the patient is considered a satisfactory result $[3,7]$. Different treatments have been proposed, including the extraction of all premolars and molars, laser ablation of inflammatory tissue, immuno-suppressive chronic treatments (cyclosporine, corticosteroids, etc...), administration of anti-inflammatory drugs (NSAIDs or corticosteroids), interferon, antibiotics and other medications. Nevertheless, none of these therapies has proven to be effective and decisive in all patients: this suggests that it is necessary to evaluate patient's symptoms and owner's wishes carefully to define an appropriate therapeutic strategy.

In order to reduce the inflammatory reaction, any tooth that, for any reason (periodontal, endodontic pathology, resorptive lesion, etc...), causes inflammation should be removed [1]. If some teeth are not removed, they must receive a strict routine professional cleaning and a thorough home hygiene in order to keep the inflammation under control. This treatment plan is often rejected because the majority of patients feel pain during the hygiene practices due to the widespread inflammation. Therefore, the most successful long-term therapy in cats with chronic gingivostomatitis has proved to be the complete extraction of all premolars and molars and a careful balancing of the alveolar bone $[2,8]$. The removal of the residual periodontal ligament, with a curette or a coarse diamond bur, also increases the chances of therapeutic success of the surgery [7]. Extraction of canines and incisors is recommended whenever inflammation extends to include the surrounding gingiva [7,9]. In conclusion, in case of significant oral inflammation, some authors recommend complete teeth extraction while others prefer not to extract the canines. Postoperative radiographic inspection is needed to confirm complete extraction of the teeth [7-9]. Surgical therapy has proven to be resolutive in most cases, although sometimes the addition of antibiotic therapy is needed, or applications of chlorhexidine $0.1-0.2 \%$ for two weeks after surgery. A further improvement of healing can occur upon dietary foods, used to favor nutritional recovery during convalescence [10]. Regardless of the choice of either teeth extraction procedure, some patients have only partial improvement and, although at much lower doses than before surgery, require long-term medical management. Fortunately, these patients are the minority [8] and correspond to subjects with long-lasting chronic inflammation and treated with high doses of glucocorticoids over long time [7,8]. Although the existing literature does not show studies with significant positive results, for many years laser ablation of the inflamed gingiva has been reported as a technique to control inflammation associated to caudal stomatitis. Some authors [11] reported an improvement after laser surgery, albeit after radical extraction of teeth. In some subjects, laser surgery yielded no substantial advantage and, often, the subjects showed transient hyperlgesia due to treatment.

If owners refuse surgery, bacterial load and inflammation can be reduced by medical therapy. In general, the treatment protocol consists in oral administration twice a day, which is quite difficult in cats, especially in subjects with pain symptoms. Furthermore, most of 
the products cause unwanted side effects. Cat owners should also be advised that long-lasting medical therapy makes cats less responsive to surgical extraction, thus predisposing to further long term medical therapy. In addition, the complete effectiveness of medical protocols has not been demonstrated. They are only able to temporarily reduce the clinical symptoms [9]. Before starting medical therapy, it is still advisable to perform professional dental prophylaxis, in order to decrease bacterial load, and complete radiographic examination of the oral cavity, followed by extractions of all sick teeth [8].

Medical therapy is based on the use of antibiotics, antiinflammatory drugs, cyclosporine, feline interferon and other products.

1. Systemic antibiotics can induce some improvement in reducing bacterial load and, therefore, oral inflammation. However, this improvement is usually temporary and often the majority of patients relapse even in the course of antibiotic therapy [7,9]. Clavulanic acid associated with amoxicillin, clindamycin and metronidazole are commonly used in the course of stomatitis.

2. Corticosteroids are the most commonly used anti-inflammatory drugs in stomatitis. The immune modulation provided by these steroids gives clinical improvement in the short term and their use is often associated with the administration of antibiotics. The long-term administration of corticosteroids can have harmful effects, such as the induction of diabetes mellitus and opportunistic infections $[9,12]$. When the use of corticosteroids is decided, it is preferable to administer the lowest effective dose and periodically monitor blood parameters (blood cell count and biochemical values). At first drugs are frequently administered parenterally (methylprednisolone $10-20 \mathrm{mg} / \mathrm{subject} / \mathrm{SC}$ ) because of pain. As soon as possible, it is preferable to switch to oral therapy, which may be suspended immediately in the event of unwanted side effects. Clinical improvement usually occurs within $24-48 \mathrm{~h}$ and can last up to 3-6 weeks. Unfortunately, disease-free intervals tend to shorten progressively and, in many cases, any treatment become ineffective. For this reason, steroid therapy should be performed only as a last option, when the cat owner does not agree with other therapeutic strategies [1].

3. Non-Steroidal Anti-Inflammatory Drugs (NSAIDs) can represent another choice. They can be used in the short term to reduce inflammation and to improve the clinical status of patients in preparation to surgery. To note that long-term NSAID therapy exposes cats to remarkable risk, of which the owner should be informed. Before starting this treatment, assessment of renal function by haemato-chemical parameters is needed. Then, degree of hydration, urinary and haemato-chemical parameters must be monitored.

4. Cyclosporine A has been proposed as immunosuppressive therapy in cats with resorptive lesion [7], especially when a post-extraction medical management is necessary: this drug was shown to be effective in cases refractory to extractions [13], while it is not a good alternative to surgery. When cyclosporin A is proposed as an alternative to long-term steroid therapy, it should be used with caution, especially in cats with hepatic or renal impairment. Moreover, the owners should be warned that there are reports of fatal opportunistic infections associated with the use of cyclosporine A [14].
5. The omega interferon has been proposed for its antiviral and immunomodulatory effects [15]. Although some studies have shown its efficacy in cases resistant to conventional treatments [16], there is no evidence that the use of omega interferon can be successfully used as a mono-therapy for the control of resorptive lesion.

6. Other drugs have been used with occasional success, although none of these therapies is currently recommended: gold salts (1-2 mg per week for 8 weeks and then monthly) [3]; lactoferrin, or lactotransferrin (applied topically $40 \mathrm{mg} / \mathrm{kg}$ ) [3,17,18]; levamisole (2-5 mg/kg PO 3 times a week) [3]; doxycycline (2 mg/kg BID, decreasing the dosage according to the effectiveness) [9]; and coenzyme Q10 (30-60 mg SID) [7].

In summary, a therapy of choice and standardized treatment for stomatitis management has not yet identified. The tools currently used are invasive (surgery) or can cause side effects (drugs) and the degree of effectiveness is variable and uncertain. Therefore, it is important to look for new therapeutic strategies to effectively counteract inflammation and improve symptoms.

Laser therapy (not to be confused with laser surgery and tissue ablation) has been applied with significant results in the management of human patients affected by Oral Mucositis (OM). Laser radiation, mostly in the red - Near Infrared (NIR) range of wavelengths, resulted effective in reducing inflammation, pain, severity and duration of OM ulcers [19-21].

A study carried out on a mouse model of OM showed that laser therapy remarkably improved and accelerated the healing process. Histological analysis on biopsies from laser-treated and untreated (control group) mice with OM showed a thinner inflammatory infiltrate in the treated animals, improved healing of the lesions and recovery of tissue architecture in comparison with untreated controls [22]. Moreover, it has been reported that red-IR laser radiation can inhibit the growth of microorganisms and increase their mortality [23-25]; such effects may be beneficial in treating disorders such as mucositis, in which the microbial load has a significant role.

An in vitro study aimed at investigating the effects of a dualwavelength NIR laser emission on the proteomic profile of muscle cells demonstrated that laser treatment increased the production of NLRP10 [26], a protein that reduces inflammatory response by inhibiting the activity of PYCARD and caspase-1, both associated with the inflammasome $[27,28]$. In addition, NIR laser radiation enhanced the expression of proteins involved in cell metabolism and structure, indicating that cellular response to irradiation was characterized by anabolic events [26]. The induction of such effects may be relevant for reduction of inflammation and improvement of tissue repair.

The above findings support the hypothesis that NIR laser therapy could be usefully applied to treat cat mucositis. The present paper reports the results of a very preliminary proof-of-concept clinical study aimed at verifying this hypothesis. Ten cats affected by stomatitis were treated by a dual wavelength NIR laser (MLS Multiwave Locked System Laser). The animals were divided into two groups, in order to compare two treatments differing for energy density. The effect of NIR laser application was assessed by clinical controls and analysis of biopsies collected from the animals before and after the cycle of laser therapy. 


\section{Materials and Methods}

\section{Subjects included in the study}

Before inclusion of patients in the study, the protocol was explained in detail to the owners. After obtaining their informed consent, animals with symptoms of stomatitis underwent the following procedures:

1. Clinical examination under sedation/anesthesia to confirm clinically the diagnosis of stomatitis. In case the diagnosis of stomatitis (involving the caudal area of the oral cavity, with or without inflammation in the remaining oral mucosa) was confirmed:

2. A complete radiographic study of the oral cavity to determine the presence of sick teeth, dental root remnants, periodontal disease, resorptive lesion or other radiologically detectable diseases.

3. Biopsy followed by histological analysis and Immunofluorescence microscopy in order to evaluate inflammation. Biopsies were performed with a scalpel. The samples obtained (diameter $\sim 5$ millimeters) included gingiva and mucosa.

Cats recently (time frame $\geq 20$ days) treated with therapies based on corticosteroids, antibiotics, NSAIDs, or combination of these drugs, and cats with systemic diseases were excluded from the study. Finally, 10 animals were enrolled. Data from the enrolled patients were collected in clinical records indicating: symptoms, medical history and therapeutic medical history (medical and surgical therapies previously attempted).

After extraction of pathological teeth, the enrolled patients underwent a therapeutic program based on treatment with a dual wavelength NIR laser system (MLS laser).

During each session of laser therapy, carried out on patients in the waking state, clinical controls were performed and the owners were interviewed on daily symptoms, with particular reference to food intake, yawn and mantle care. At the end of therapeutic cycle, a clinical examination and a second biopsy, followed by histological analysis and Immunofluorescence microscopy, were performed under sedation/anesthesia in order to evaluate inflammation and the effect of therapy.
The treatment protocol applied in this study did not alter the procedures normally applied to patients with FCGS Type 2 (involving the caudal area of the oral cavity, with or without inflammation in the remaining oral mucosa). The application of the laser therapy did not prevent the use of pharmacological therapies at any time they deemed necessary. The sampling of the biopsies that were used for histology was part of the standardized diagnostic protocol, regardless of the application of laser therapy.

\section{Laser therapy}

Details of the laser source have been previously described [26]. Briefly, the treatments were performed with a Multiwave Locked System laser (MLS laser, ASA Srl, Vicenza, Italy), a commercial laser source built in compliance with EC/EU rules, which received FDA approval and is widely used in clinics and veterinary medicine. MLS laser is a class IV NIR laser with two synchronized sources (laser diodes). The two sources have different wavelength, peak power and emission mode. The first one is a pulsed $905 \mathrm{~nm}$ laser diode with 25 $\mathrm{W}$ peak optical power. The pulse frequency may be varied in the range $1-2000 \mathrm{~Hz}$, thus varying the average power delivered to the tissue. The second laser diode $(808 \mathrm{~nm})$ may operate in continuous (power $1.1 \mathrm{~W}$ ) or frequenced (repetition rate $1-2000 \mathrm{~Hz}, 550 \mathrm{~mW}$ mean optical power) mode, with a $50 \%$ duty ratio independently of the repetition rate. The two sources emit radiation synchronously and the propagation axes of the two laser beams are coincident.

Laser treatment was administered using a hand piece held a few millimeters away from the tissue, with a spot diameter of $2 \mathrm{~cm}$. Each treatment session consisted in irradiating six areas (the size of each area corresponding to the spot) within the oral cavity (6 points/ session): isthmus mouth, upper arch at the IV premolar, lower arch at the I molar, both left and right, as shown in figure 2 .

Animals were divided randomly into two groups, as follows:

Group I ( $\mathrm{n}=5$ patients) received laser therapy with the following parameters:

- 2 treatments per week for 5 consecutive weeks (10 treatments)

- Duty cycle: fixed $\delta 50 \%$

- Frequency: $36 \mathrm{~Hz}$

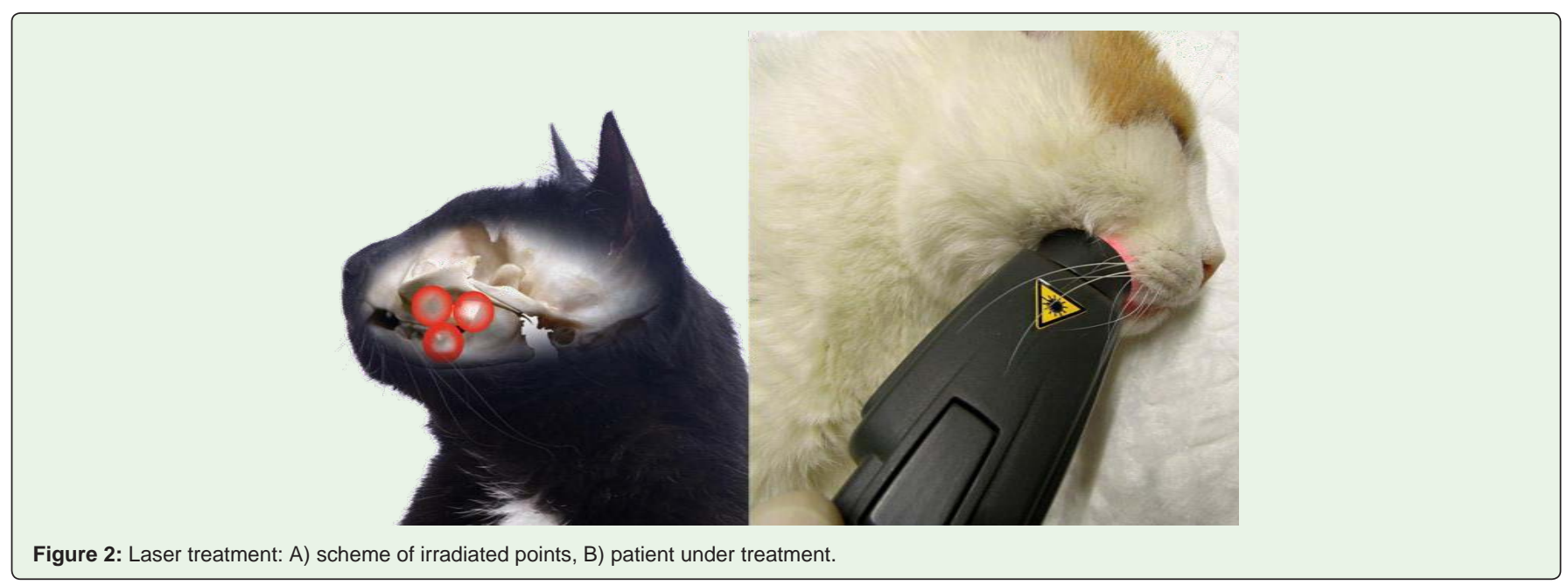

Citation: Squarzoni P, Bani D, Cialdai F and Monici M. NIR Laser Therapy 
- Exposure time/treated point: $8 \mathrm{sec}$

- Energy/point: $1 \mathrm{~J}$

- Fluence: $0.32 \mathrm{~J} / \mathrm{cm}^{2}$

Group II $(n=5)$ received laser therapy with the following parameters:

- 2 treatments per week for 5 consecutive weeks (10 treatments)

- Duty cycle: fixed $\delta 50 \%$

- Frequency: $36 \mathrm{~Hz}$

- Exposure time/treated point: $4 \mathrm{sec}$

- Energy/point: $0.5 \mathrm{~J}$

- Fluence: $0.16 \mathrm{~J} / \mathrm{cm}^{2}$

\section{Clinical data}

During the first visit, in addition to biopsy, clinical and radiographic examination, data from medical history were collected to assess:

- dysphagia

- algic events during meal and yawn

- reluctance to take food

- anorexia (full or partial)

- opaque mantle

These parameters were also investigated during every session of laser therapy and a score from 0 (absence of symptoms) to 3 (very intense symptoms) was assigned to each patient. The scoring criteria used for clinical data is reported in table 1.

\section{Histology}

Small fragments of oral mucosa were surgically removed from clinically affected areas before and after laser therapy. They were fixed in $4 \%$ formaldehyde in $0.1 \mathrm{M}$ phosphate-buffer, $\mathrm{pH} 7.4$, dehydrated in graded ethanol and embedded in paraffin for light microscopic analysis. Histological sections, $6 \mu \mathrm{m}$ thick, stained with hematoxylin $\&$ eosin were examined and photographed under a Reichert Microstar IV light microscope equipped with an Eurekam 9 high-resolution videocamera (BEL Engineering, Monza, Italy) interfaced with a PC by a dedicated software (BEL view, BEL Engineering).

Each specimen was subjected to semi-quantitative evaluation of the histopathological changes involving the surface epithelium, the inflammatory infiltrate and the blood vessels in the lamina

Table 1: Clinical data scoring criteria.

\begin{tabular}{|c|c|c|c|c|}
\hline Scoring & Dysphagia & Food Intake & Yawn & Licking \\
\hline 0 & Absent & Normal & Normal & Normal \\
\hline 1 & Mild & $\begin{array}{c}\text { With some } \\
\text { difficulty } \\
\text { With serious } \\
\text { difficulty }\end{array}$ & $\begin{array}{c}\text { With some } \\
\text { difficulty } \\
\text { With serious } \\
\text { difficulty }\end{array}$ & $\begin{array}{c}\text { With some } \\
\text { difficulty }\end{array}$ \\
\hline 2 & Moderate & $\begin{array}{c}\text { With serious } \\
\text { difficulty }\end{array}$ \\
\hline 3 & Severe & $\begin{array}{c}\text { Inability to take } \\
\text { food }\end{array}$ & Inability to yawn & Inability to lick \\
\hline
\end{tabular}

propria. The scoring criteria used are reported in table 2. Scoring was performed on at least 3 consecutive microscopical fields examined at a $x 400$ final magnification.

\section{Immunofluorescence microscopy}

Immunofluorescence microscopy was performed on tissue sections obtained from biopsies collected before and after laser therapy (see 2.4). After dewaxing and rehydration, to prevent nonspecific antibody reactions, specimens were immersed in $3 \%$ bovine serum albumin (BSA, Sigma, Steinheim, Germany) in PBS for $30 \mathrm{~min}$. Then, Fluorescein Isothiocyanate (FITC)-conjugated antiCD3 antibody (1:100 in PBS $0.5 \%$ BSA) was applied directly to the specimens overnight at $4^{\circ} \mathrm{C}$.

The CD3 T-cell co-receptor is part of the TCR (T-cell receptor) complex, which generates an activation signal in T lymphocytes. CD3 is a transmembrane protein, bound to the membranes of all mature T-cells. Being highly specific, CD3 is a reliable marker for T-cells in tissues [29].

Samples were evaluated using an epifluorescence microscope (Eclipse TE, Nikon, Florence, Italy) at 20x magnifications and imaged by a HiRes IV digital CCD camera (DTA, Pisa, Italy).

Based on the CCD images, a quantification of Immunofluorescence intensity was carried out by image analysis routines (Image J analysis program) applied on control and treated samples. After appropriate thresholding to eliminate background signal and subsequent image masking, a pixel intensity histogram was acquired. The mean histogram value was divided by the region of interest area in pixel^ ${ }^{2}$ to obtain a mean Immunofluorescence density value.

\section{Statistical analysis}

Data were compared using Student $\mathrm{t}$ tests, as appropriate. Statistical analyses were conducted assuming the conventional significance level of $5 \%(\mathrm{p}<0.05)$. Calculations were performed using Microsoft Excel (Microsoft Office Home and Business 2013).

\section{Results}

\section{Clinical data}

Before laser therapy, all the examined subjects showed dysphagia, algic events during meals and often during the yawn, reluctance to take food, weight loss and opaque mantle. The score related to clinical data of the 10 patients is shown in table 3 and means of the data are reported in figure 3. Evaluation was assessed at Initial, Intermediate (after $5^{\text {th }}$ treatment), Final and Follow-up phase of the study.

At the final control, made in connection with the last treatment $\left(10^{\text {th }}\right)$, in animals of Group I, laser therapy (Protocol I) induced a slight/moderate improvement of symptoms.

Table 2: Histopathological scoring criteria.

\begin{tabular}{|l|c|c|c|}
\hline \multicolumn{1}{|c|}{ Severity } & $\begin{array}{c}\text { Surface } \\
\text { Epithelium }\end{array}$ & $\begin{array}{c}\text { Inflammatory } \\
\text { Infiltrate }\end{array}$ & $\begin{array}{c}\text { Blood } \\
\text { Vessels }\end{array}$ \\
\hline$+\quad$ normal or slight & Normal & Focal & Normal \\
\hline++ mild & Thinned & $\begin{array}{c}\text { Sub epithelial, } \\
\text { band-like }\end{array}$ & Dilated \\
\hline++ severe & Ulcerated & Full thickness & -- \\
\hline
\end{tabular}




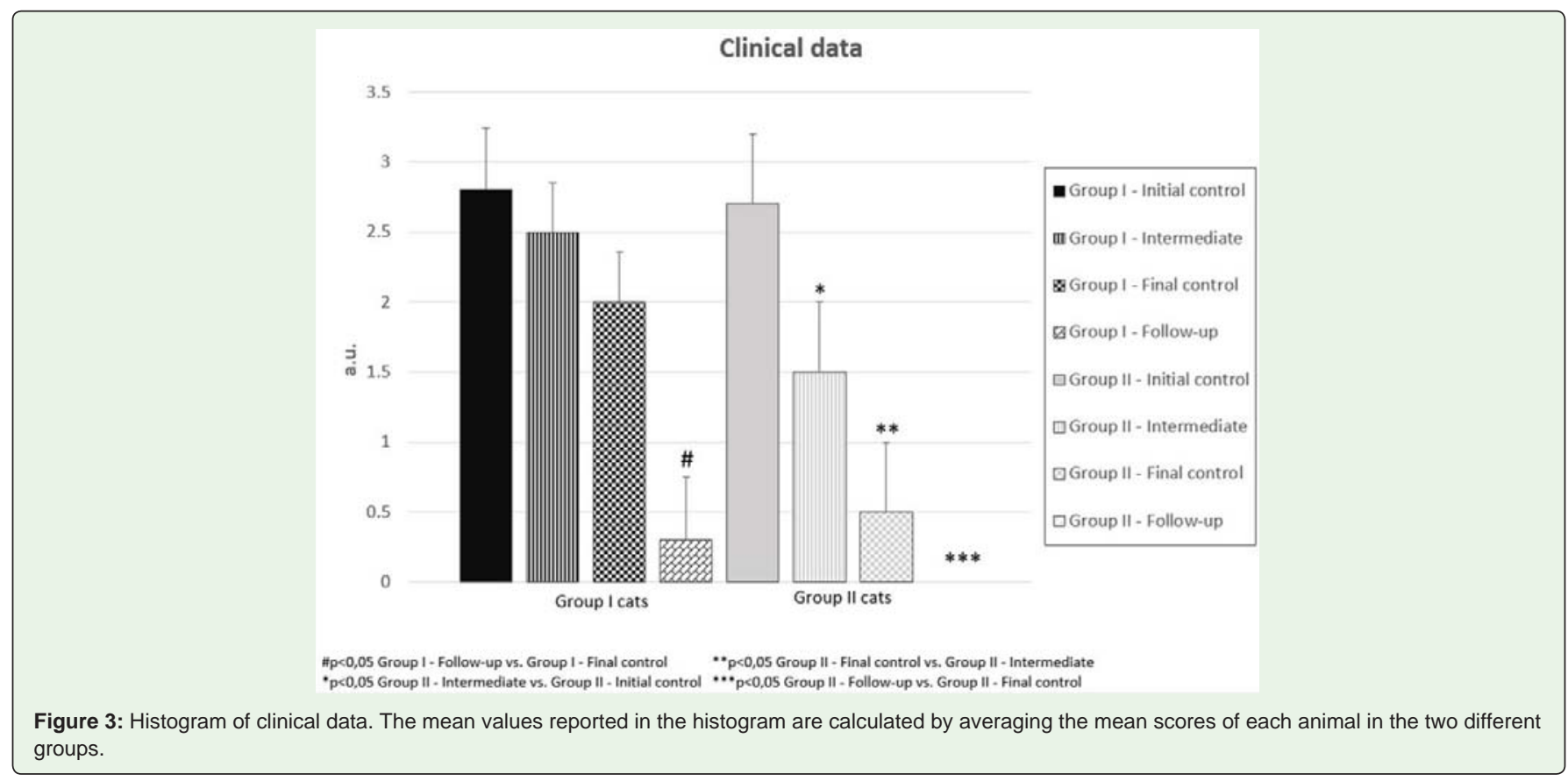

On the contrary, laser therapy (Protocol II) was very effective in treating animals of Group II, which showed a significant improvement of symptomatology, often observed as early as the fifth/

Table 3: Patient's clinical course. sixth treatment. In one case, complete recovery was observed at the medical examination performed at the last session of laser therapy (final control) (Figure 4). However, after 5-6 months by the end of treatment, a significant improvement of symptomatology was reported both in Group I and Group II.

\begin{tabular}{|c|c|c|c|c|c|c|c|c|c|c|c|c|c|c|c|c|c|c|c|c|}
\hline \multicolumn{21}{|c|}{ Group I } \\
\hline & \multicolumn{5}{|c|}{ Initial control } & \multicolumn{5}{|c|}{ Intermediate } & \multicolumn{5}{|c|}{$\begin{array}{c}\text { Final Control } \\
\text { (end of the therapy) }\end{array}$} & \multicolumn{5}{|c|}{$\begin{array}{l}\text { Follow-up (5-6 months by } \\
\text { the end of the therapy) }\end{array}$} \\
\hline & $\begin{array}{l}\frac{\pi}{\bar{T}} \\
\frac{\pi}{\frac{\pi}{0}} \\
\frac{0}{0} \\
\frac{0}{0}\end{array}$ & 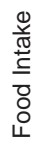 & 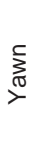 & 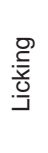 & 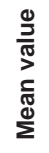 & $\frac{\frac{\pi}{7}}{\frac{\pi}{2}}$ & 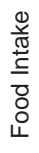 & 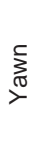 & 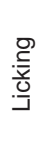 & 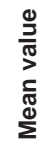 & $\begin{array}{l}\frac{\pi}{7} \\
\frac{\pi}{\frac{\pi}{0}} \\
\frac{0}{2} \\
\frac{0}{0}\end{array}$ & 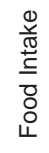 & 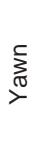 & 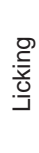 & 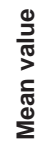 & $\begin{array}{l}\frac{\pi}{2} \\
\frac{\pi}{2} \\
\frac{0}{0} \\
\text { के }\end{array}$ & 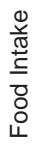 & 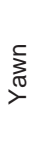 & $\frac{0}{\stackrel{0}{\frac{D}{0}}}$ & 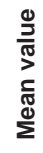 \\
\hline Case 1 & 3 & 2 & 2 & 1 & 2 & 2 & 3 & 2 & 1 & 2 & 1 & 1 & 2 & 2 & 1.5 & 0 & 0 & 0 & 0 & 0 \\
\hline Case 2 & 3 & 3 & 3 & 3 & 3 & 3 & 3 & 2 & 2 & 2.5 & 2 & 2 & 2 & 2 & 2 & 0 & 0 & 1 & 1 & 0.5 \\
\hline Case 3 & 3 & 3 & 3 & 3 & 3 & 3 & 3 & 3 & 3 & 3 & 3 & 3 & 2 & 2 & 2.5 & 0 & 1 & 0 & 1 & 0.5 \\
\hline Case 4 & 3 & 3 & 3 & 3 & 3 & 2 & 2 & 3 & 3 & 2.5 & 2 & 2 & 2 & 2 & 2 & 0 & 0 & 0 & 0 & 0 \\
\hline Case 5 & 3 & 3 & 3 & 3 & 3 & 3 & 3 & 2 & 2 & 2.5 & 2 & 2 & 2 & 2 & 2 & 1 & 1 & 0 & 0 & 0.5 \\
\hline \multicolumn{21}{|c|}{ Group II } \\
\hline & \multicolumn{5}{|c|}{ Initial control } & \multicolumn{5}{|c|}{ Intermediate } & \multicolumn{5}{|c|}{$\begin{array}{c}\text { Final Control } \\
\text { (end of the therapy) }\end{array}$} & \multicolumn{5}{|c|}{$\begin{array}{c}\text { Follow-up } \\
\text { (5-6 months by the end of the } \\
\text { therapy) }\end{array}$} \\
\hline & $\begin{array}{l}\frac{\pi}{5} \\
\frac{\pi}{0} \\
\frac{0}{0} \\
\frac{0}{0}\end{array}$ & 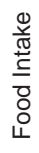 & 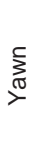 & 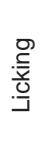 & $\begin{array}{l}\frac{0}{\frac{0}{\sigma}} \\
\frac{\pi}{2} \\
\frac{\sigma}{0} \\
\sum\end{array}$ & $\begin{array}{l}\frac{\pi}{7} \\
\frac{\pi}{\frac{\pi}{2}} \\
\frac{0}{0} \\
\frac{0}{0}\end{array}$ & 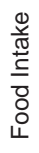 & 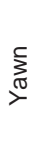 & 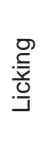 & $\begin{array}{l}\frac{0}{J} \\
\frac{\pi}{\pi} \\
\frac{5}{\sigma} \\
\Sigma\end{array}$ & $\begin{array}{l}\frac{\pi}{7} \\
\frac{\pi}{\frac{\pi}{2}} \\
\frac{0}{2} \\
\frac{0}{0}\end{array}$ & 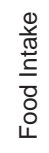 & 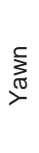 & 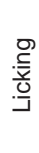 & 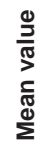 & $\begin{array}{l}\frac{\pi}{2} \\
\frac{\pi}{0} \\
\frac{0}{0} \\
\frac{0}{0}\end{array}$ & 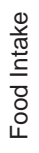 & 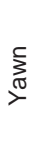 & 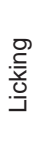 & 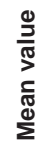 \\
\hline Case 1 & 3 & 3 & 3 & 3 & 3 & 2 & 2 & 3 & 1 & 2 & 1 & 1 & 1 & 1 & 1 & 0 & 0 & 0 & 0 & 0 \\
\hline Case 2 & 3 & 2 & 3 & 2 & 2.5 & 1 & 1 & 2 & 0 & 1 & 0 & 0 & 0 & 0 & 0 & 0 & 0 & 0 & 0 & 0 \\
\hline Case 3 & 3 & 3 & 3 & 3 & 3 & 2 & 2 & 1 & 1 & 1.5 & 0 & 0 & 1 & 1 & 0.5 & 0 & 0 & 0 & 0 & 0 \\
\hline Case 4 & 3 & 3 & 1 & 1 & 2 & 2 & 1 & 1 & 0 & 1 & 0 & 0 & 0 & 0 & 0 & 0 & 0 & 0 & 0 & 0 \\
\hline Case 5 & 3 & 3 & 3 & 3 & 3 & 2 & 3 & 1 & 2 & 2 & 1 & 1 & 1 & 1 & 1 & 0 & 0 & 0 & 0 & 0 \\
\hline
\end{tabular}




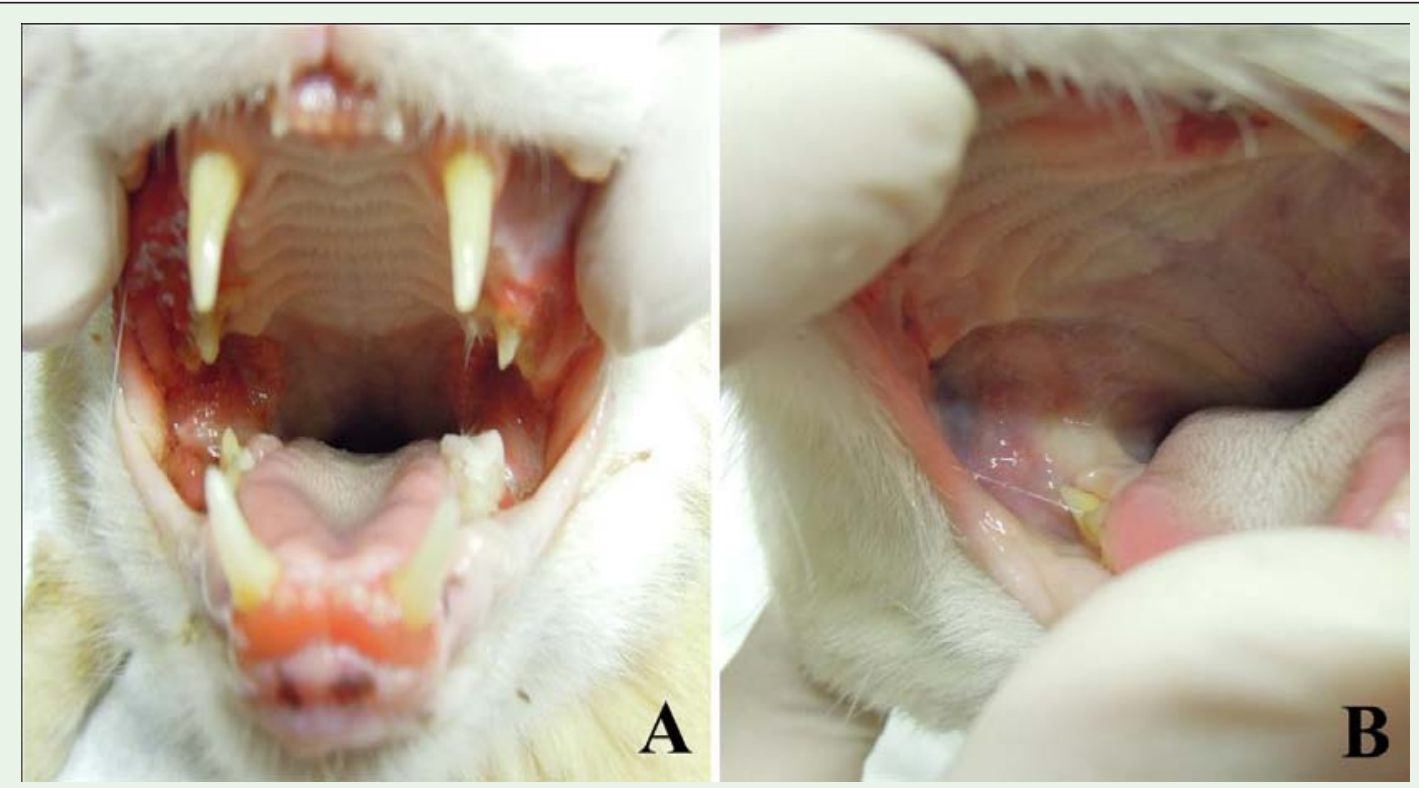

Figure 4: Pictures of a patient (Group II) collected before (A) and after laser therapy (B). At the end of the therapy, the complete recovery is evident.

\section{Histological analysis}

Examination of the samples of oral mucosa collected before the treatment period from the animals of Group I showed the occurrence of mild to severe histopathological alterations, consisting in thinning and ulceration of large tracts of the surface epithelium and the presence of an extended inflammatory infiltrate, mainly composed of lymphocytes and mononuclear cells featuring monocytes/ macrophages (Figure 5A). Laser therapy (Protocol I) did not result in significant changes of these histopathological features, as revealed by the biopsies taken at the end of the treatments (Figure 5B). Semiquantitative scoring confirmed the visual observations (Table 3 and Figure 6). A slight reduction of the inflammatory infiltrate was only observed in 2 out of 5 cats.

Histological analysis of oral mucosa samples collected before the treatment period from the animals of Group II showed mild to severe histopathological alterations very similar to those above described in the Group I cats (Figure 5C). In Group II, however, laser therapy (Protocol II) caused a substantial improvement of the histopathological features (Figure 5D), mainly consisting in recovery of surface epithelial integrity and marked reduction of the inflammatory infiltrate in the lamina propria, which nearly disappeared in 2 of the 5 cats. Semi-quantitative scoring confirmed the visual observations (Table 4 and Figure 6).

\section{Immunofluorescence microscopy}

Immunofluorescence microscopy was performed on tissue sections obtained from biopsies collected from all the subjects enrolled in the study before and after laser therapy. The tissue sections were incubated with FITC-conjugated anti-CD3 antibodies (see 2.5) to evaluate the presence of T-lymphocytes in the inflamed tissues before and after laser therapy. In fact, T-lymphocytes have a prominent role in regulating the evolution of inflammation towards chronicization [30]. The tissue sections from all the biopsies

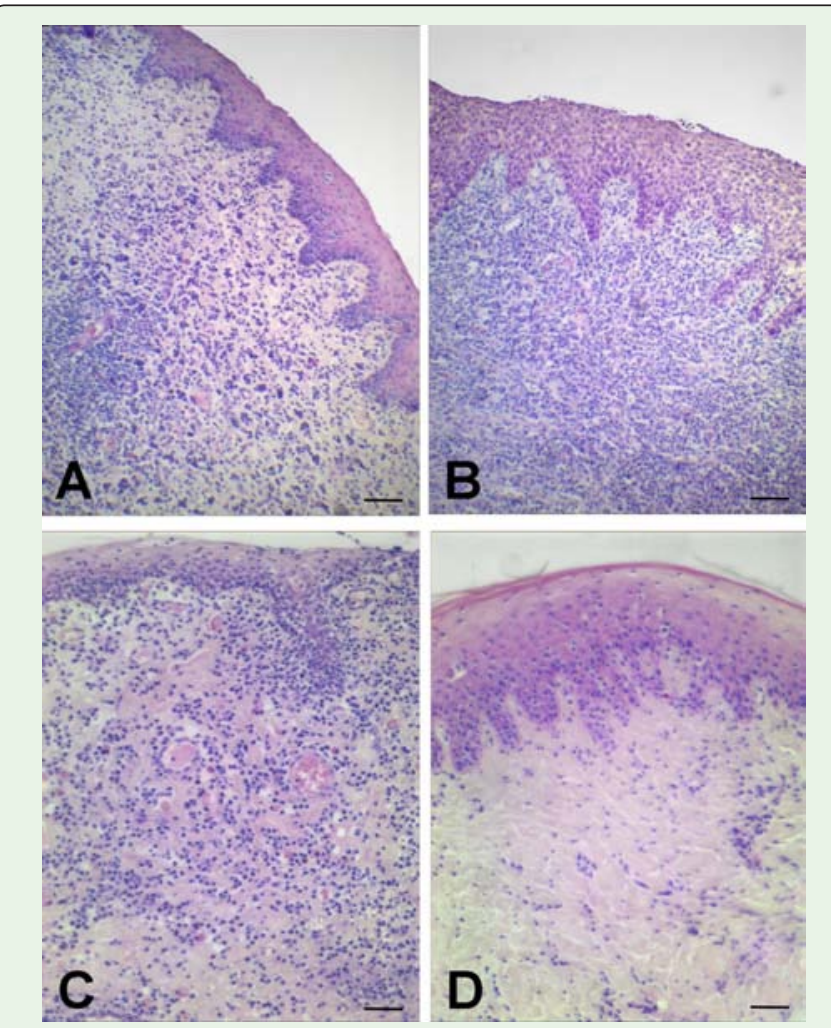

Figure 5: Representative histopathological features of oral mucosal biopsies from cats of the different groups. A) Group I, pre-treatment; B) Group I, laser treatment at high dose $\left(0.32 \mathrm{~J} / \mathrm{cm}^{2}\right)$; C) Group II, pre-treatment; D) Group II, laser treatment at low dose $\left(0.16 \mathrm{~J} / \mathrm{cm}^{2}\right)$. Before the treatments, the lamina propria of the oral mucosa showed a dense inflammatory infiltrate, chiefly composed of lymphoid and monocytoid cells, blood vessel dilatation and thickening of the squamous epithelium. High-dose laser treatment caused no appreciable reduction of the inflammatory infiltrate, whereas the low-dose laser treatment did. Hematoxylin \& Eosinstaining. Bar $=100 \mu \mathrm{m}$. 
Table 4: Histopathological scoring.

\begin{tabular}{|c|c|c|c|c|c|c|}
\hline \multicolumn{7}{|c|}{ Group I cats } \\
\hline \multirow{2}{*}{$\begin{array}{c}\text { Animal } \\
\text { No }\end{array}$} & \multicolumn{3}{|c|}{ Pre-laser therapy } & \multicolumn{3}{|c|}{ Post-laser therapy } \\
\hline & epithelium & Inflammatory infiltrate & Blood vessels & epithelium & Inflammatory infiltrate & Blood vessels \\
\hline 1 & + & $+/++$ & ++ & + & + & ++ \\
\hline 2 & $++/+++$ & +++ & ++ & ++ & +++ & $+/++$ \\
\hline 3 & $++/+++$ & +++ & ++ & $++/+++$ & +++ & ++ \\
\hline 4 & $++/+++$ & +++ & ++ & $+/++$ & + & $+/++$ \\
\hline 5 & $++/+++$ & ++ & ++ & $+/+++$ & ++ & ++ \\
\hline \multicolumn{7}{|c|}{ Group II cats } \\
\hline \multirow{2}{*}{$\begin{array}{c}\text { Animal } \\
\text { No }\end{array}$} & \multicolumn{3}{|c|}{ Pre-laser therapy } & \multicolumn{3}{|c|}{ Post-laser therapy } \\
\hline & epithelium & Inflammatory infiltrate & Blood vessels & epithelium & Inflammatory infiltrate & Blood vessels \\
\hline 1 & +++ & +++ & ++ & + & $+/++$ & ++ \\
\hline 2 & ++ & +++ & + & + & - & ++ \\
\hline 3 & +++ & $+++/++$ & ++ & + & $+/++$ & ++ \\
\hline 4 & $++/+$ & $++/+$ & + & + & - & + \\
\hline 5 & +++ & $+++/++$ & ++ & ++ & $++/+$ & + \\
\hline
\end{tabular}

\section{Histopathological scoring}

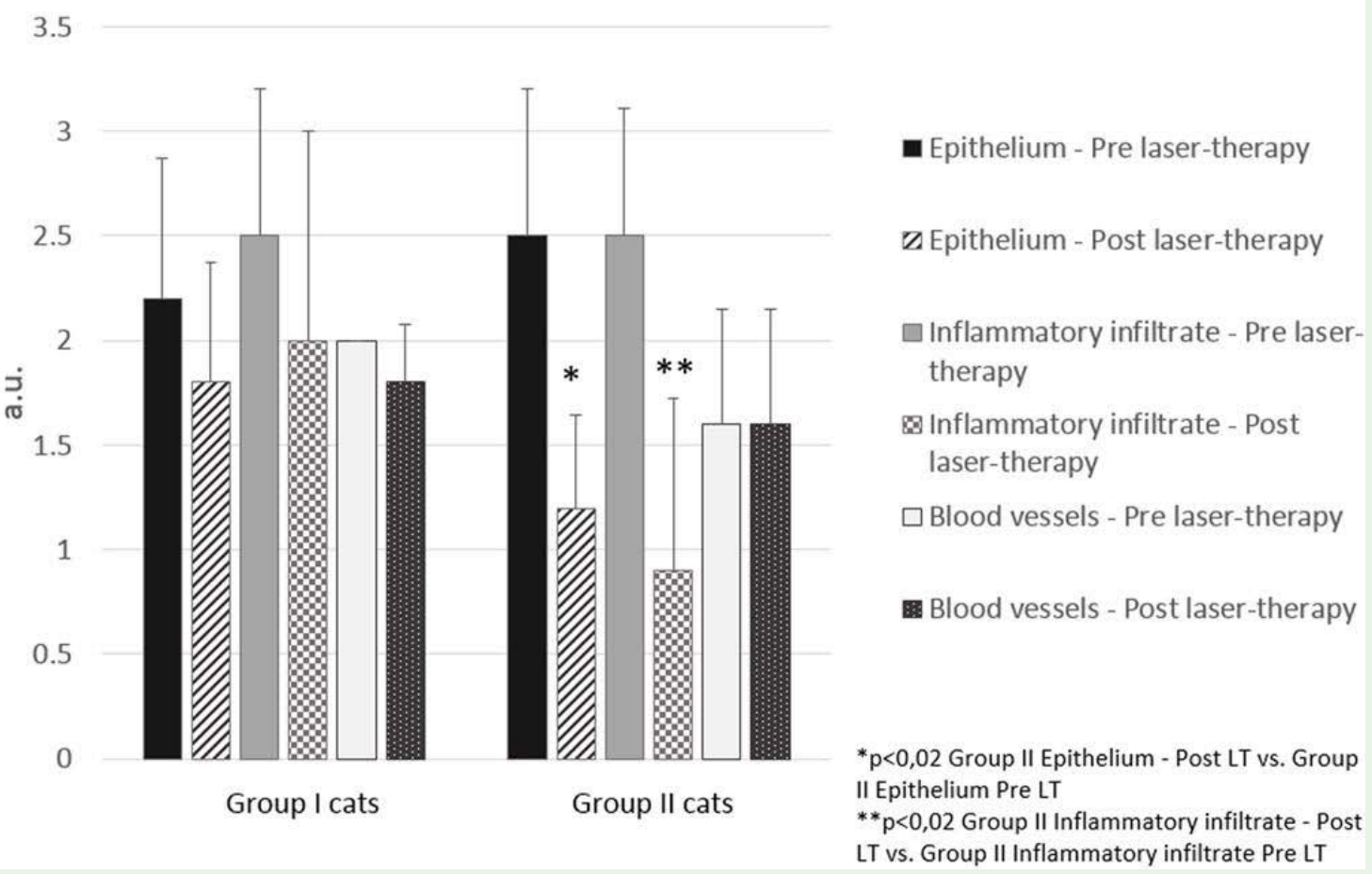

Figure 6: Histogram of histopathological scoring. The mean values reported in the histogram are calculated by averaging the scores of each animal in the two different groups. 


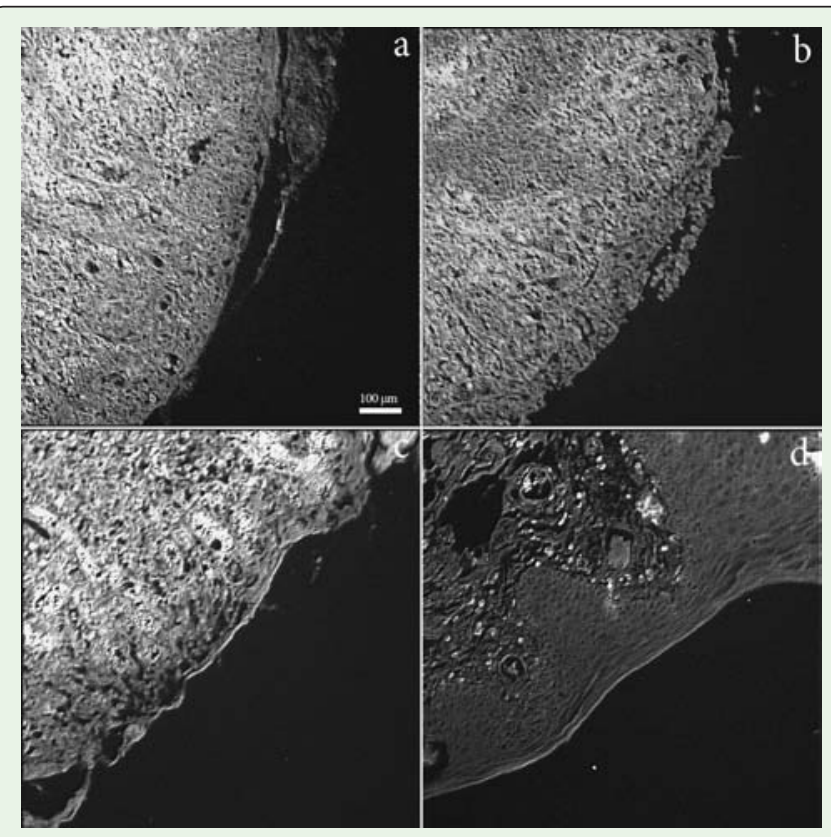

Figure 7: Immunofluorescence analysis of CD3 positive cells in specimens of oral mucosa from cats of the different groups. A) Group I, pre-treatment; B) Group I, laser treatment at high dose $\left(0.32 \mathrm{~J} / \mathrm{cm}^{2}\right)$; C) Group II, pretreatment; D) Group II, laser treatment at low dose $\left(0.16 \mathrm{~J} / \mathrm{cm}^{2}\right)$. In agreement with histology, the tissue sections from biopsies collected before laser therapy showed areas with high density of $\mathrm{T}$ lymphocytes (Figs. 4A and $4 C$ ) and evident alterations of the epithelium. Low-dose laser treatment significantly decreases T-lymphocytes and partially restores the epithelium.

(Groups I and II) collected before laser therapy showed areas with a high density of T lymphocytes (Figures 7A and 7C) and marked alterations of the epithelium, in agreement with histology. After
Table 5: Immunofluorescence analysis: quantification of immunofluorescence intensity.

\begin{tabular}{|c|c|c|}
\hline \multicolumn{3}{|c|}{ Group I cats } \\
\hline Animal No & Pre laser-therapy & Post laser-therapy \\
\hline 1 & 108 & 95 \\
\hline 2 & 91 & 103 \\
\hline 3 & 115 & 93 \\
\hline 4 & 122 & 97 \\
\hline 5 & 126 & 101 \\
\hline mean & 112,4 & 97,8 \\
\hline S.D & 13,8 & 4,1 \\
\hline \multicolumn{3}{|c|}{ Group II cats } \\
\hline Animal No & Pre laser-therapy & Post laser-therapy \\
\hline 1 & 100 & 38 \\
\hline 2 & 93 & 28 \\
\hline 3 & 78 & 52 \\
\hline 4 & 146 & 71 \\
\hline 5 & 124 & 69 \\
\hline mean & 108,2 & $51,6^{\star}$ \\
\hline S.D. & 26,9 & 18,8 \\
\hline
\end{tabular}

laser therapy, the appearance of the samples obtained from Group I did not differ significantly from that of pre-therapy specimens (Figure 7B). On the contrary, in tissue sections collected from Group II T-lymphocytes significantly decreased and the epithelium was partially restored (Figure 7D). Quantification of Immunofluorescence intensity confirmed the visual observations (Table 5 and Figure 8).

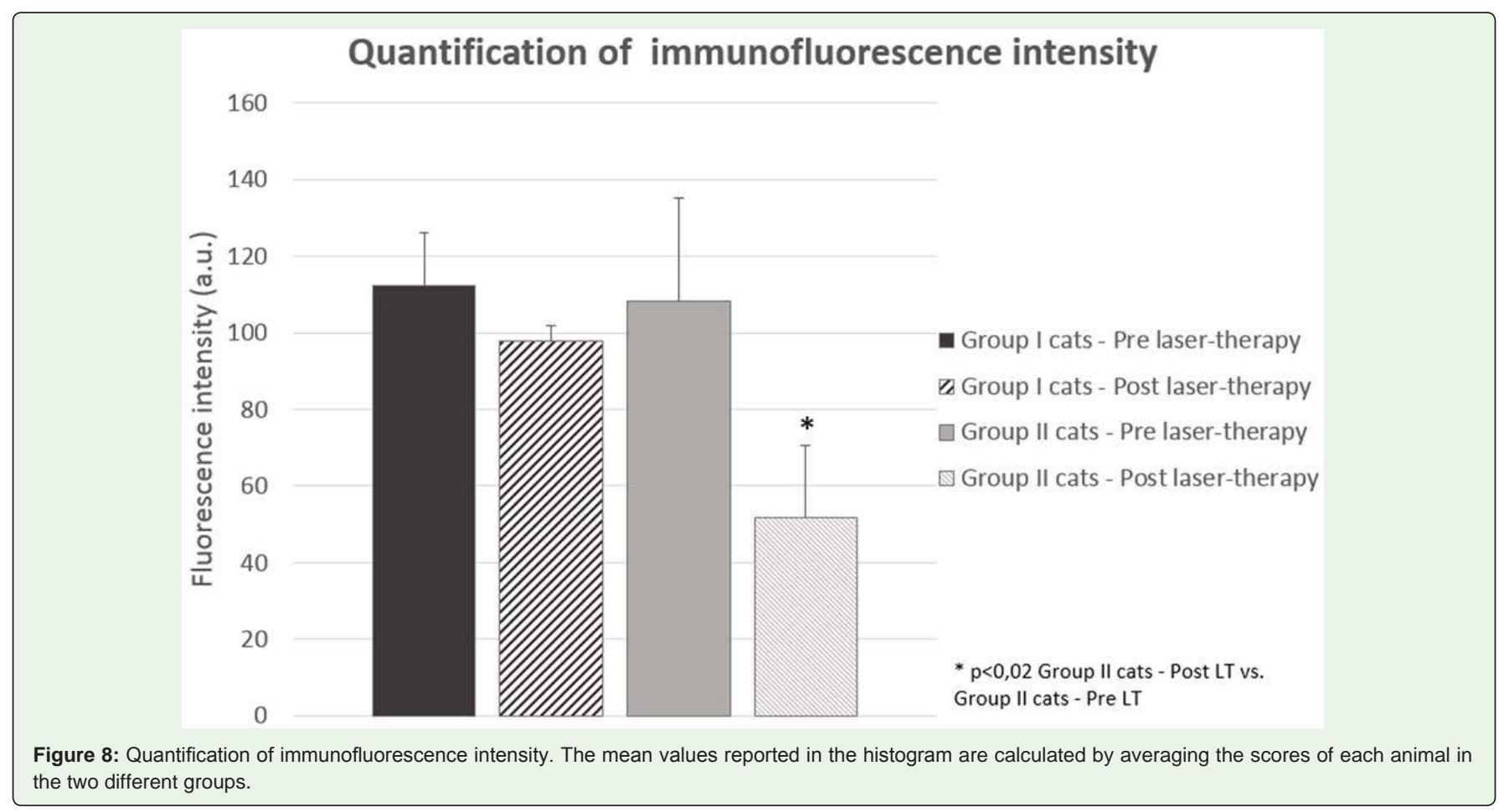

Citation: Squarzoni P, Bani D, Cialdai F and Monici M. NIR Laser Therapy 


\section{Discussion and Conclusions}

In patients with FCGS (feline chronic gingivo-stomatitis) type 2, the goal of therapy is to reduce and possibly eliminate inflammation, although achieving this goal is often very difficult $[3,7]$. None of the treatments proposed up to date, including extraction of all premolars and molars, has been proved effective and decisive in all patients, thus prompting the search for new therapeutic strategies. In this study, the possibility to apply NIR laser therapy for treating FCGS type 2 was evaluated and the effectiveness of two protocols, differing for the administered energy density (fluence), was assessed. At the end of treatment sessions, from the clinical point of view, Group I cats, treated with protocol I (fluence $0.32 \mathrm{~J} / \mathrm{cm}^{2}$, energy/point $1 \mathrm{~J}$ ), showed a feeble response to the therapy ranging from no effect to moderate improvement of symptoms. Consistently, histology revealed a sporadic, modest improvement in the amount of inflammatory infiltrate, with no significant changes in the appearance of the surface epithelium and blood vessels in the lamina propria. Confirming histological data, Immunofluorescence microscopy showed high infiltration of $\mathrm{T}$ lymphocytes in the lamina propria, both before and after therapy, when a slight, not significant decrease of infiltrating $\mathrm{T}$ lymphocytes was observed. However, as mentioned above, the clinical symptoms improved during the weeks following the course of laser therapy and after 5-6 months by the end of the treatment the improvement became significant.

On the contrary, the Protocol II (fluence $0.16 \mathrm{~J} / \mathrm{cm}^{2}$, energy/ point $0.5 \mathrm{~J}$ ) applied to the patients belonging to the Group II and characterized by a lower energy dose, induced a significant improvement, as assessed by the regression of symptoms as well as by histology and Immunofluorescence microscopy. In 4 of the 5 patients, the improvement was already significant after the fifth treatment. In Group II, histology of tissue sections obtained after laser therapy showed, in comparison with pre-therapy sections, marked decrease in the inflammatory infiltrate and recovery of a nearly normal appearance of the surface epithelium. The persistence of a variable degree of vasodilation, despite the improvement of other parameters, might be due to laser treatment. It is well known that NIR laser therapy induces a moderate vasodilation which is useful in favoring the wash-out of pro-inflammatory cytokines and improving the oxygenation of diseased tissues [31,32]. Immunofluorescence microscopy, performed to evaluate the presence of $\mathrm{CD} 3$-positive $\mathrm{T}$ lymphocytes, revealed a strong decrease of $\mathrm{T}$ lymphocytes in sections from biopsies collected after laser therapy (Protocol II), in keeping with the decrease in inflammatory infiltrate shown by histology. The finding that the treatment Protocol II decreased inflammation and induced tissue restoring agrees with the results of previous studies, carried out on cells irradiated with the same laser source used in this study, which demonstrated the increase of NLRP 10 protein, known as inflammasome inhibitor, and over expression of proteins involved in anabolic processes [26].

The marked difference between the therapeutic effects of Protocol I and II is clearly due to the different fluence applied. Protocol I, with higher fluence (and total energy released) than Protocol II, possibly led to excess vasodilation and cell stimulation, resulting in a paradoxical pro-inflammatory response (at least in the short term) which takes place in already inflamed tissues, as occurs in FCGS type 2. This scenario fits well with the poor effectiveness of Protocol I in the short term and induction of delayed benefits. On the contrary, the lower fluence used in Protocol II allowed to exploit the positive effects of the laser therapy since early stages of treatment, avoiding to exacerbate the evolution of the inflammatory process. Once again, these results prove that the effectiveness of laser therapy strongly depends on treatment parameters, which should be carefully chosen and validated for application to different types of patients and diseases.

In conclusion, the outcomes of this preliminary study demonstrate that laser therapy with suitable treatment parameters can be effective in the management of FCGS type 2. This result is in agreement with previous reports on the application of laser therapy to oral mucositis in humans $[21,33,34]$. Although it is difficult to compare feline and human mucositis, since the two diseases probably have different causes, in both cases laser therapy is effective to alleviate similar symptoms, namely inflammation, lesions of the oral mucosa, pain and difficult feeding.

In the management of FCGS, laser therapy has shown the following clinical advantages:

- Easy application: in the protocols used in this study, the timing of application is reduced to a few seconds and the number of sessions is limited; these factors favor cooperation of patients and full acceptance by owners.

- No known side effects.

- Virtually instantaneous analgesic effect.

- It can be used as exclusive or complementary treatment of the disease. Moreover, it can be used to control pain when scheduling surgery (extraction of all the premolars and molars, etc.).

- Full compatibility with other therapeutic treatments in progress.

The limit of this study is the small number of patients and a follow up limited to a few months. Therefore, at this preliminary stage of research, it is not possible to predict if FCGS can be completely controlled or healed by laser therapy.

However, from a clinical point of view, the most part of subjects undergoing laser therapy, even if with different response times, showed an improvement of the symptoms and, in particular, a better food intake, less pain during the yawn and increased licking of the mantle.

The present results support the need to extend the study, involving a higher number of patients, and to explore further laser irradiation modes in order to develop more effective therapeutic protocols.

\section{References}

1. Camy G, Fahrenkrug P, Gracis M. Proposed guidelines on the management of feline chronic gingivostomatitis (FCGS) syndrome: A consensus statement. Consultation version September $19^{\text {th }}$ European Congress of Veterinary Dentistry (ECVD), Nice, September 23-25, 2010.

2. Lyon KF. Gingivostomatitis. Vet Clin North Am Small Anim Pract. 2005; 35: 891-911.

3. Wiggs RB, Lobprise HB. Domestic feline oral and dental disease. In: Veterinary Dentistry, Principles and Practice. Philadelphia: Lippincott-Raven, USA. 1997; 482-517. 
4. Lommer MJ, Verstraete FJM. Concurrent oral shedding of feline calicivirus and feline herpesvirus 1 in cats with chronic gingivostomatitis. Oral Microbiol Immunol. 2003; 18: 131-134.

5. Hardy Jr WD, Zuckerman E, Corbishley J. Serological evidence that Bartonella cause gingivitis and stomatitis in cats. In: American Veterinary Dental Society Meeting, Savannah, GA 2002.

6. Niemiec BA. Veterinary Periodontology. John Wiley \& Sons, Inc. 2013; 95

7. Debowes LJ. Problems with the gingiva. In: Small Animal Dental, Oral and Maxillofacial Disease, a Color Handbook (Niemiec BA ed.). London: Manson. 2010; 159-181.

8. Niemiec BA. Oral pathology. Top Companion Anim Med. 2008; 23: 59-71.

9. Bellows J. Treatment of oropharyngeal inflammation. In: Feline Dentistry: Oral Assessment, Treatment, and Preventative Care. Ames IA: Wiley-Blackwell, 2010; 242-268.

10. Theyse LF, Logan EI, Picavet P. Partial extraction in cats with gingivitis stomatitis-pharyngitis-complex-Beneficial effects of a recovery food. In: Livro de Resumos Hill's European Symposium on Oral Care, Amesterdão 2003 64-65.

11. Lewis JR, Tsugawa AJ, Reiter AM. Use of $\mathrm{CO}_{2}$ laser as an adjunctive treatment for caudal stomatitis in a cat. J Vet Dent. 2007; 24: 240-249.

12. Niemiec BA. Problems of the oral mucosa In: Small Animal Dental, Ora and Maxillofacial Disease, a Color Handbook (Niemiec, B.A. ed.). London: Manson. 2010; 183-198.

13. Lommer MJ. Use of cyclosporine for the treatment of refractory feline gingivostomatitis. In: Proceedings of the $22^{\text {nd }}$ Annual Dental Forum. 2008.

14. Beatty J, Barra V. Acute toxoplasmosis in two cats on cyclosporine therapy. Aust Vet J. 2003; 81: 339

15. Southerden P, Gorrel C. Treatment of a case of refractory feline chronic gingivostomatitis with recombinant interferon omega. J Small Anim Pract. 2007; 48: 104-106.

16. Hennet PR, Camy GA, McGahie DM, Albouy MV. Comparative efficacy of a recombinant feline interferon omega in refractory cases of calicivirus-positive cats with caudal stomatitis: a randomised, multi-centre, controlled, doubleblind study in 39 cats. J Feline Med Surg. 2011; 13: 577-587.

17. Sato R, Inanami O, Tanaka Y, Takase M, Naito Y. Oral administration of bovine lactoferrin for treatment of intractable stomatitis in feline immunodeficiency virus (FIV)-positive and FIV-negative cats. Am J Vet Res. 1996; 57: 14431446.

18. Addie DD, Radford A, Yam PS, Taylor DJ. Cessation of feline calicivirus shedding coincident with resolution of chronic gingivostomatitis in a cat. $J$ Small Anim Pract. 2003; 44: 172-176.

19. Gobbo M, Ottaviani G, Perinetti G, Ciriello F, Beorchia A, Giacca M, et al. Evaluation of nutritional status in head and neck radio-treated patients affected by oral mucositis: efficacy of class IV laser therapy. Support Care Cancer. 2014; 22: 1851-1856.

20. Maver-Biscanin M, Mravak-Stipetic M, Jerolimov V. Effect of low-level lase therapy on Candida albicans growth in patients with denture stomatitis. Photomed Laser Surg. 2005; 23: 328-332.
21. Chermetz M, Gobbo M, Ronfani L, Ottaviani G, Zanazzo GA, Verzegnassi $\mathrm{F}$, et al. Class IV laser therapy as treatment for chemotherapy-induced ora mucositis in onco-haematological paediatric patients: a prospective study. Int J Paediatr Dent. 2014; 24: 441-449

22. Ottaviani G, Gobbo M, Sturnega M, Martinelli V, Mano M, Zanconati F, et al. Effect of class IV laser therapy on chemotherapy-induced oral mucositis: a clinical and experimental study. Am J Pathol. 2013; 183: 1747-1757.

23. Basso FG, Oliveira CF, Fontana A, Kurachi C, Bagnato VS, Spolidório DM, et al. In Vitro effect of low-level laser therapy on typical oral microbial biofilms. Braz Dent J. 2011; 22: 502-510.

24. Souza RC, Junqueira JC, Rossoni RD, Pereira CA, Munin E, Jorge AO. Comparison of the photodynamic fungicidal efficacy of methylene blue, toluidine blue, malachite green and low-power laser irradiation alone against Candida albicans. Lasers Med Sci. 2010; 25: 385-389.

25. Bornstein E, Hermans W, Gridley S, Manni J. Near-infrared photoinactivation of bacteria and fungi at physiologic temperatures. Photochem Photobiol. 2009; 85: 1364-1374.

26. Monici M, Cialdai F, Ranaldi F, Paoli P, Boscaro F, Moneti G, et al. Effect of IR laser on myoblasts: a proteomic study. Mol Biosyst. 2013; 9: 1147-1161.

27. Wang $Y$, Hasegawa M, Imamura R, Kinoshita T, Kondo C, Konaka K, et al. PYNOD, a novel Apaf-1/ CED4-like protein is an inhibitor of ASC and caspase-1. Int Immunol. 2004; 16: 777-786.

28. Imamura R, Wang $\mathrm{Y}$, Kinoshita $\mathrm{T}$, Suzuki M, Noda $\mathrm{T}$, Sagara J, et al. Antiinflammatory activity of PYNOD and its mechanism in humans and mice. J Immunol. 2010; 184: 5874-5884.

29. Chetty R, Gatter K. CD3: structure, function, and role of immunostaining in clinical practice. J Pathol. 1994; 173: 303-307.

30. Ross R. The role of T lymphocytes in inflammation. Proc Natl Acad Sci U S A. 1994; 91: 2879.

31. Fukuda TY, Tanji MM, Silva SR, Sato MN, Plapler H. Infrared low-level diode laser on inflammatory process modulation in mice: pro- and anti-inflammatory cytokines. Lasers Med Sci. 2013; 28: 1305-1313.

32. Schaffer M, Bonel H, Sroka R, Schaffer PM, Busch M, Reiser M, et al. Effects of $780 \mathrm{~nm}$ diode laser irradiation on blood microcirculation: preliminary findings on time-dependent T1-weighted contrast-enhanced Magnetic Resonance Imaging (MRI). J Photochem Photobiol B. 2000; 54: 55-60.

33. Jaguar GC, Prado JD, Nishimoto IN, Pinheiro MC, de Castro DO Jr, da Cruz Perez DE, et al. Low-energy laser therapy for prevention of oral mucositis in hematopoietic stem cell transplantation. Oral Dis. 2007; 13: 538-543.

34. Carvalho PA, Jaguar GC, Pellizzon AC, Prado JD, Lopes RN, Alves FA. Evaluation of low-level laser therapy in the prevention and treatment of radiation-induced mucositis: a double-blind randomized study in head and neck cancer patients. Oral Oncol. 2011; 47: 1176-1181. 